\title{
Evaluation serum levels of Insulin Growth Factor-1 (IGF-1) and its association with clinical parameters in severe COVID-19
}

\author{
Parisa Feizollahi ${ }^{1}$. Somaieh matin ${ }^{2,3} \cdot$ Seyed Askar Roghani ${ }^{1,4}$. Shayan Mostafaei ${ }^{5}$. Elham Safarzadeh ${ }^{6}$ \\ Mahdi Taghadosi ${ }^{7}$ (1)
}

Received: 2 October 2021 / Accepted: 30 November 2021 / Published online: 31 January 2022

(c) The Author(s), under exclusive licence to Springer Nature Switzerland AG 2021

\begin{abstract}
Background Severe coronavirus disease-2019 (COVID-19) is associated with dysregulated immune response and extreme inflammatory injury. Considering the role of insulin growth factor-1 (IGF-1) in immune-mediated and inflammatory reactions, this study was conducted to investigate the IGF-1 contribution to the pathogenesis of severe form of COVID-19.

Material and methods Sixty-two patients with severe COVID-19 and 52 healthy subjects were enrolled in this study. The serum levels of IGF-1 were measured using a solid-phase enzyme-linked chemiluminescent immunoassay on an Immulite 2000 system (Siemens Healthcare Diagnostics.

Result The serum levels of IGF-1 had no significant difference in COVID-19 patients compared to the healthy subjects $(p=0.359)$. There was a positive correlation between IGF-1 and age in the severe COVID-19 patients, while a negative correlation was observed for the serum levels of IGF-1 and age in the control group $(r=0.364, p=0.036, r=-0.536, p=0.001$, respectively). Moreover, IGF-1 was remarkably associated with hypertension, neurogenic disease, shock, and nausea in patients with the severe form of COVID-19 ( $p=0.031, p=0.044, p=0.01, p=0.03$, respectively).

Conclusion Our results pointed to the complex role of IGF-1 in the severe form of COVID-19, and its association with clinical parameters, and some risk factors in the severe form of COVID-19.
\end{abstract}

Keywords IGF- $1 \cdot$ Severe COVID-19 $\cdot$ Age $\cdot$ Clinical parameters

$\begin{array}{ll}\text { Abbreviations } \\ \text { COVID-19 } & \text { Severe coronavirus disease-2019 } \\ \text { IGF-1 } & \text { Insulin growth factor-1 }\end{array}$

SARS-CoV-2 Severe acute respiratory syndrome-coronavirus 2

ALI Acute lung injury

MOF Multi-organ failure

Parisa Feizollahi and Somaieh matin contributed equally to this work.

Elham Safarzadeh

E.safarzadehe@arums.ac.ir

Mahdi Taghadosi

mtaghad@gmail.com

1 Immunology Department, Faculty of Medicine, Kermanshah University of Medical Sciences, Kermanshah, Iran

2 Department of Internal Medicine, Emam Khomeini Hospital, Ardabil University of Medical Sciences, Ardabil, Iran

3 Gastrointestinal and Liver Disease Research Center, Razi Hospital, Guilan University of Medical Sciences, Rasht, Iran

$\begin{array}{ll}\text { IGF2R } & \begin{array}{l}\text { Insulin-like growth factor II receptor } \\ \text { Phosphatidylinositol-3-kinases/protein } \\ \text { kinase B }\end{array} \\ \text { MAPK } & \begin{array}{l}\text { Mitogen-activated protein kinase } \\ \text { ARDS }\end{array} \\ \text { Acute respiratory disease syndrome } \\ \text { WHO } & \text { World Health Organization } \\ \text { BPD } & \text { Body mass index } \\ \text { PR } & \text { Blood pressure diastolic } \\ \text { RR } & \text { Pulse rate } \\ & \text { Respiratory rate }\end{array}$

4 Medical Biology Research Center, Health Technology Institute, Kermanshah University of Medical Sciences, Kermanshah, Iran

5 Division of Clinical Geriatrics, Department of Neurobiology, Care Sciences and Society, Karolinska Institutet, Stockholm, Sweden

6 Department of Microbiology, Parasitology, and Immunology, Ardabil University of Medical Sciences, Ardabil, Iran

7 Department of Immunology, Kermanshah University of Medical Sciences, Kermanshah, Iran 
$T$

CNS

Temperature

Central nervous system

\section{Introduction}

Coronavirus Disease 2019 (COVID-19) is an acute viral respiratory infection caused by the severe acute respiratory syndrome-coronavirus 2 (SARS-CoV-2) (Team EE 2020). COVID-19 is associated with a critical and overwhelming impact on the health system and life-threatening medical conditions, particularly pneumonia and acute lung injury (ALI) (Of the International CSG 2020). SARS-CoV-2 has flu-like symptoms ranging from asymptomatic and mild infection to severe ALI and multi-organ failure (MOF), eventually leading to death (Zhu et al. 2020). Studies have shown that the severe consequences in COVID-19 patients do not directly evolve by the replication of the SARS-CoV-2 itself but are relatively due to the destructive inflammatory responses caused by the immense activation of the immune cells. Therefore, the adjusted immune reaction is indispensable to control and eliminate SARS-CoV-2 infection. On the other hand, maladjusted immune responses and the elaboration of the massive number of inflammatory mediators and cytokines can have adverse and sometimes lethal sequelae (Li et al. 2020; Qin et al. 2020; Sinha et al. 2020).

Insulin-like growth factor 1 (IGF1) is a 70-amino acid long polypeptide hormone, belonging to the insulin-like growth factor family [insulin-like growth factor II (IGF2), growth hormone, insulin-like growth factor 1 receptor (IGF1R), insulin-like growth factor II receptor (IGF2R), and insulin-like growth factor binding protein 1-6 (IGFBP1-6)] (Jogie-Brahim et al. 2009) with several functions, including tissue growth and development, insulin-like activity, proliferation, pro-survival, anti-aging, antioxidant and neuroprotective (Martin-Estal et al. 2016). Furthermore, IGF-1 is involved in regulating the biological responses of the immune system through phosphatidylinositol-3-kinases/ protein kinase B (PI3K/AKT) and mitogen-activated protein kinase (MAPK) signaling pathways (Myers et al. 1992). Various studies have proven the role of the IGF-1 signal transduction in lung development, as well as inflammatory diseases like ALI and Acute respiratory disease syndrome (ARDS), asthma, cancer, inflammation, and fibrosis (Stiles and D'Ercole 1990; Ahasic et al. 2012; Mu et al. 2020; Narasaraju et al. 2006; He et al. 2019; Li et al. 2016). IGF-1 is also implicated in the regulation of inflammation in the immune system (DeBoer et al. 2017; Heemskerk et al. 1999). A study conducted by Hoshino et al. showed that IGF-1 mRNA expression in the bronchial cells of asthmatic patients was significantly higher than those of healthy individuals, which was remarkably associated with fibrosis in epithelial cells (Hoshino et al. 1998). Given the evidence for
Table 1 Demographic characteristics and IGF-1 serum levels between COVID-19 patients and healthy control groups

\begin{tabular}{lllll}
\hline Characteristics & Level & $\begin{array}{l}\text { COVID-19 } \\
(n=62)\end{array}$ & Control $(n=52)$ & $p$ value \\
\hline Age (years) & - & $60.19 \pm 18.56$ & $64.13 \pm 15.03$ & 0.221 \\
Sex & Male & $30(48.4 \%)$ & $28(53.8 \%)$ & 0.145 \\
BMI $\left(\mathrm{kg} / \mathrm{m}^{2}\right)$ & - & $26.84 \pm 4.24$ & $27.47 \pm 5.53$ & 0.497 \\
IGF-1 $(\mathrm{ng} / \mathrm{mL})$ & - & $83.04 \pm 51.96$ & $75.73 \pm 31.19$ & 0.359 \\
\hline
\end{tabular}

Data are expressed as mean $\pm \mathrm{SD}$ or $N(\%)$

$B M I$ body mass index, IGF-1 insulin growth factor

the role of IGF-1 in lung development and its involvement in immune responses, evaluating the IGF-1 levels may further shed light on mechanistic underlying the pathogenesis of COVID-19.

Emerging data from previous studies indicated that the clinical manifestations of COVID-19 are heterogeneous and highly depend on many underlying conditions. Senescence and comorbidities, including hypertension, diabetes, obesity, renal failure, and lung diseases, are significant conditions associated with increased risk of developing ARDS, MOF, and consequently death in patients with COVID-19. Some other variables are also worth mentioning, like ethnicity and sex (Kassir 2020).

Considering immune-mediated inflammatory responses underpinning COVID-19 pathogenesis, involvement of IGF-1 in immunological reactions, and association documented between IGF-1 and underlying conditions such as diabetes, obesity, hypertension, aging (Teppala and Shankar 2010; Spielman et al. 2014; Ounis et al. 2010; Norling et al. 2020; Delafontaine et al. 2004; Salminen and Kaarniranta 2010) this study aimed to investigate the serum levels of IGF-1 and its association with clinical parameters and underlying conditions in the severe form of COVID-19.

\section{Methods and materials}

\section{Study design and patient characteristics}

Sixty-two patients aged from 19 to 91 years $[60.19 \pm 18.56$, (mean \pm standard error of the mean)] with severe COVID-19 and 52 healthy subjects aged from 21 to $90(64.13 \pm 15.03)$ after matching for age and sex were enrolled in the current study. The diagnosis of COVID-19 was confirmed using RT-PCR (real-time reverse transcriptase-polymerase chain reaction) assay and specialist physician according to the defined clinical and other laboratory detection parameters. The demographic and clinical characteristics of both groups are presented in Table 1. The project was approved by the ethics committee of the Kermanshah and Ardabil 
University of Medical Sciences (IR.KUMS.REC.990599, and IR.ARUMS.REC.1399.562), and all defined protocols were done according to the Helsinki declaration. All participants gave written informed consent before taking part in the study.

\section{Blood sample preparation and measurements of IGF-1 serum levels}

Blood Samples were taken from the antecubital vein of patients and controls, and they were processed within $1 \mathrm{~h}$ after collection, centrifuged for $15 \mathrm{~min}$, aliquot, and stored at $-80^{\circ} \mathrm{C}$ until analysis. IGF-1 was measured in serum using a solid-phase enzyme-linked chemiluminescent immunoassay on an Immulite 2000 system (Siemens Healthcare Diagnostics, Inc).

\section{Statistical analysis}

Statistical analysis was performed with SPSS software (version 21.0, SSPS Inc., Chicago, Illinois, USA). Results were presented as mean \pm standard deviation (SD). The distribution Normality in the groups was determined by Kolmogorov-Smirnov test (K-S test). P value is based on the independent $t$ test/or Mann-Whitney nonparametric test for statistical comparisons between both groups based on the continuous variables. Categorical variables were analyzed through the chi-square/or Fisher exact test between both patients and healthy individuals. The association between two variables was determined by Spearman rank correlation. $p$ value indicated as statistically significant at the level of $<0.05$.

\section{The calculation of body mass index (BMI)}

The calculation of weight and height were done based on standard protocol and the World Health Organization (WHO). BMI is a person's weight in kilograms divided by the square of height in meters. Normal weight: 18.5-24.9, overweight: $25.0-29.9$, obese: $\geq 30$.

\section{Results}

\section{Demographic characteristics and the serum levels of IGF-1}

The demographic characteristics and quantitative determination of the IGF-1 serum concentration and the statistical evaluation of these results are presented in Table 1 . The mean BMI in patients and control groups were $26.84 \pm 4.24$ $\left(\mathrm{kg} / \mathrm{m}^{2}\right)$ and $26.84 \pm 4.24\left(\mathrm{~kg} / \mathrm{m}^{2}\right)$, respectively, in which no significant differences were found between the amounts of

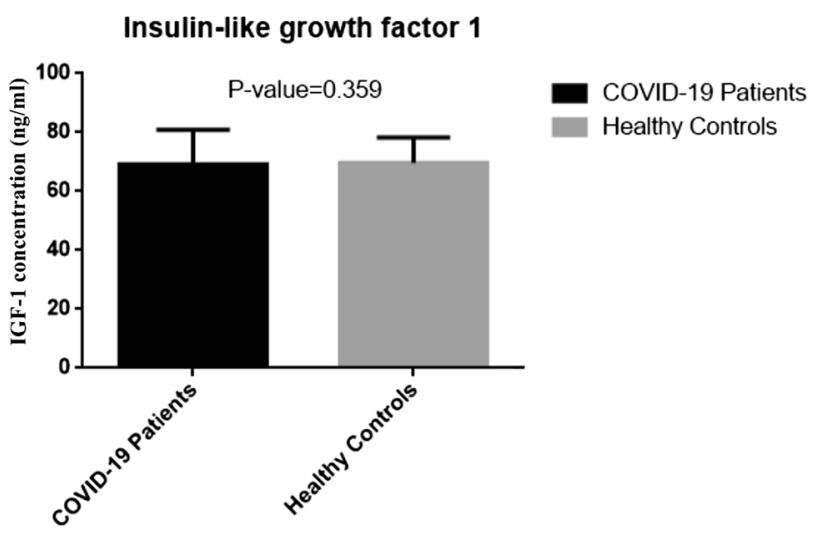

Fig. 1 The serum levels of IGF-1 in the severe patients with COVID19 and healthy subjects. There was not a significant difference for IGF-1 in severe patients with COVID-19 compared to healthy subjects using a solid-phase enzyme-linked chemiluminescent immunoassay $(p=0.359)$

BMI in studied groups (Table 1). Although the serum levels of IGF-1 in COVID-19 patients $(83.04 \pm 51.96 \mathrm{ng} / \mathrm{mL})$ was higher than that obtained in controls $(75.73 \pm 31.19 \mathrm{ng} / \mathrm{mL}$; $p=0.359$ ), the mean serum concentration of IGF-1 in the patients' group did not significantly differ from that of the healthy group (Fig. 1).

\section{Correlation matrix between IGF-1 serum levels with age and $B M I$}

The age-adjusted mean IGF-1 level was evaluated in each group. The correlation coefficients were calculated between IGF-1 levels, sex, age, and BMI to determine the IGF-1-sex, IGF-1-age, and IGF-1-BMI associations. Statically significant correlations were observed between IGF-1 levels and age in both studied groups. IGF-1 levels were positively correlated with age in COVID-19 patients $(r=0.364, p=0.036$ ). Conversely, negatively correlated with age in healthy individuals was shown $(r=-0.536, p=0.001)$ (Table 2, Figs. 2, 3 , respectively). In the regression analysis-adjusting for sex and BMI in COVID-19 patient, the relationship was non-significant, $(r=0.082, p=0.444 r=0.046, p=0.725$, respectively). Similar results were observed for the relationship between IGF-1 with sex and BMI in healthy control (Table 2).

\section{Association of the IGF-1 serum levels and clinical characteristics in COVID-19 patients}

We next investigated the relationship between IGF-1 level and clinical characteristics of patients. Among co-morbidity in patients with COVID-19, IGF-1 was significantly associated with hypertension $(p=0.031)$, Neurogenic diseases $(p=0.044)$, shock $(p=0.01)$, and Nausea $(p=0.03)$ 
Table 2 Correlation matrix between IGF-1serum levels with age and BMI in each group

\begin{tabular}{llll}
\hline Group & Variable & Coefficient $(r)$ & $p$ value \\
\hline COVID-19 patients & Age & 0.364 & $\mathbf{0 . 0 3 6}$ \\
& BMI & 0.046 & 0.725 \\
& Sex & 0.082 & 0.444 \\
Healthy subjects & Age & -0.536 & $\mathbf{0 . 0 0 1}$ \\
& BMI & 0.014 & 0.921 \\
& Sex & 0.180 & 0.121 \\
\hline
\end{tabular}

The given data showed a positive correlation between the serum IGF-1 levels and age in patients with severe COVID-19 and a remarkable negative correlation between IGF-1 levels and the age of healthy controls. There was no significant difference for IGF-1 serum levels with BMI and sex in the severe patients with COVID-19 and the control group. $B M I$ body mass index. $p$ value was considered statistically significant at the level of $<0.05$

(Table 3). Moreover, no significant correlation matrix was observed between IGF-1 levels with blood pressure, blood pressure diastolic (BPD), pulse rate (PR), respiratory rate (RR), and temperature $(T)$ in the severe COVID-19 patients (Table 4).

\section{Discussion}

The majority of patients with COVID-19 have a selflimiting infection and recover, but in some cases, it leads to severe symptoms and death (Azkur et al. 2020). It is worth mentioning, the abruptly worsening conditions of these patients are mainly due to systematic inflammatory assault caused by the uncontrolled or dysregulated immune responses, considered as the cytokine storm, leading to ARDS and multiple organ failure (Ye et al. 2020). To the best of our knowledge, this study is the first to assess the serum levels of IGF-1 in severe COVID19 patients compared to healthy individuals. Our results showed that the serum levels of IGF-1 in severe COVID-19 patients did not differ significantly compared to healthy subjects.

Previous studies have revealed controversial results regarding the impact of IGF-1 on lung pathology. The research performed by Krein PM et al. showed the increased IGF-1 immunostaining in lung biopsy specimens from patients with fibro-proliferative ARDS (Krein et al. 2003). Interestingly, elevated IGF-1 levels also have been reported in the bronchoalveolar lavage (BAL) fluid of patients with early ARDS (Schnapp et al. 2006). Besides, IGF-1 contributed to Influenza A virus-mediated acute inflammatory lung injury, which can be considered a novel approach to influenza treatment ( $\mathrm{Li}$ et al. 2019).

Conversely, reduced levels of IGF-1 have been found in ARDS cases compared to the control group with no

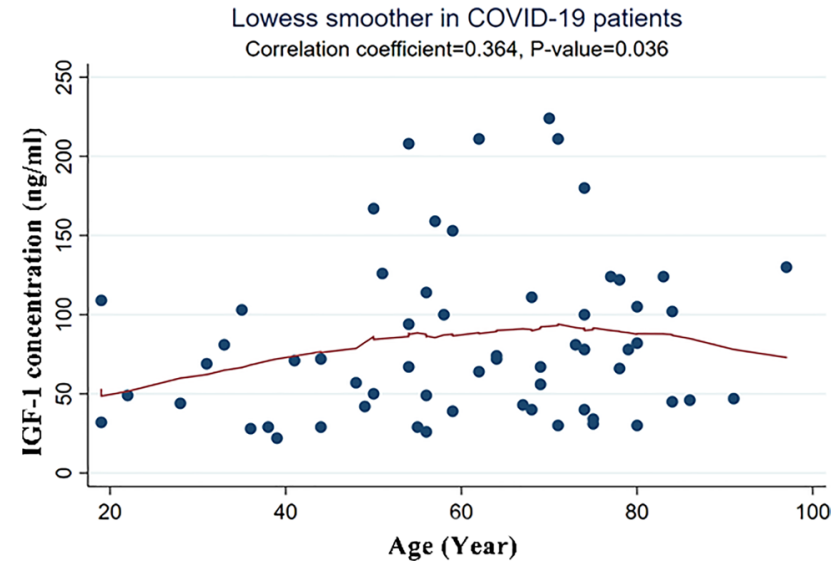

Fig. 2 Correlation of IGF-1 serum levels and age in the patients with COVID-19. The provided graph depicts a significant positive correlation between the serum IGF-1 levels and age in severe patients with COVID-19 using the LOWESS smoothing method $(r=0.364$, $p=0.036$ )

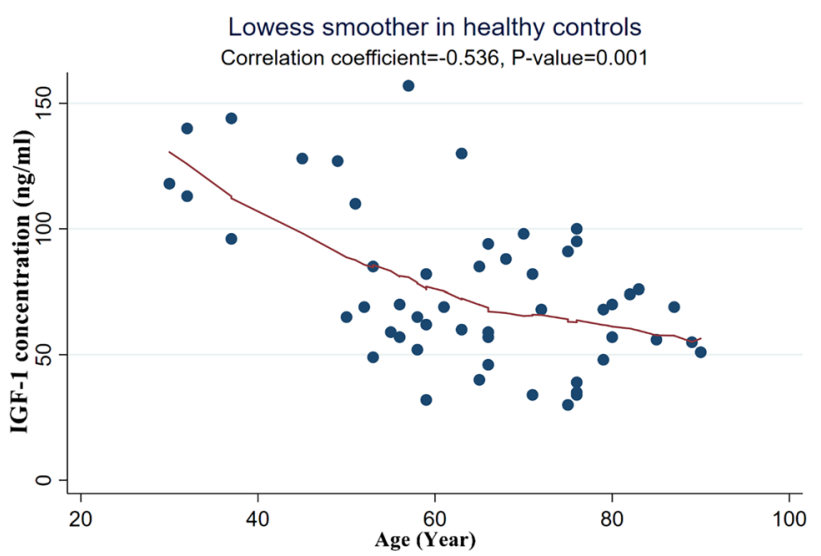

Fig. 3 Correlation of IGF-1 serum levels and age in the healthy subjects. The shown graph depicts a remarkable negative correlation between IGF-1 levels and age of healthy controls using the LOWESS smoothing method $(r=-0.536, p=0.001)$

risk of ARDS, which is positively associated with mortality among ARDS cases (Ahasic et al. 2012). A retrospective study conducted by Xikang Fan et al. demonstrated a significant reduction in IGF-1 and IGFBP3 levels in the non-survivor patients with COVID-19, which were mainly older than survivors. For instance, the IGF-1 serum levels in the COVID-19 patients with a mean age of $70.55 \pm 7.72$ was 4.79-17.09 (nmol/L), while they were 24.53-58.00 (nmol/L) in COVID-19 patients with a mean age of $65.11 \pm 9.34$. Also, in the study mentioned above, a positive correlation between COVID-19 mortality and IGF-1 further supported the role of the IGF pathway in COVID19-related ARDS (Fan et al. 2020). 
Table 3 Association between IGF-1 levels and clinical characteristics of 62 severe COVID-19 patients

\begin{tabular}{|c|c|c|c|}
\hline Characteristics & Level & IGF-1 & $p$ value \\
\hline \multirow[t]{2}{*}{ Hypertension } & Negative & $89.17 \pm 56.43$ & 0.031 \\
\hline & Positive & $64.26 \pm 28.87$ & \\
\hline \multirow[t]{2}{*}{ CKD } & Negative & $85.54 \pm 53.58$ & 0.338 \\
\hline & Positive & $66.50 \pm 38.19$ & \\
\hline \multirow[t]{2}{*}{ IHD } & Negative & $86.14 \pm 59.99$ & 0.605 \\
\hline & Positive & $79.14 \pm 40.43$ & \\
\hline \multirow[t]{2}{*}{ Neurogenic diseases } & Negative & $82.40 \pm 52.15$ & 0.044 \\
\hline & Positive & $122.0 \pm 44.17$ & \\
\hline \multirow[t]{2}{*}{ Diabetic Mellitus } & Negative & $83.60 \pm 52.68$ & 0.899 \\
\hline & Positive & $81.72 \pm 51.66$ & \\
\hline \multirow[t]{2}{*}{ Affected person } & Negative & $80.33 \pm 49.50$ & 0.361 \\
\hline & Positive & $96.90 \pm 64.28$ & \\
\hline \multirow[t]{2}{*}{ Exposure history } & Negative & $80.51 \pm 51.09$ & 0.489 \\
\hline & Positive & $91.57 \pm 55.90$ & \\
\hline \multirow[t]{2}{*}{ Fever } & Negative & $79.0 \pm 48.18$ & 0.663 \\
\hline & Positive & $85.17 \pm 54.31$ & \\
\hline \multirow[t]{2}{*}{ Vomiting } & Negative & $85.89 \pm 56.27$ & 0.459 \\
\hline & Positive & $74.33 \pm 35.85$ & \\
\hline \multirow[t]{2}{*}{ Abdomen pain } & Negative & $83.62 \pm 54.17$ & 0.858 \\
\hline & Positive & $87.25 \pm 41.86$ & \\
\hline \multirow[t]{2}{*}{ Rhinorrhea } & Negative & $83.06 \pm 54.34$ & 0.988 \\
\hline & Positive & $82.5 \pm 55.86$ & \\
\hline \multirow[t]{2}{*}{ Cough } & Negative & $81.47 \pm 55.97$ & 0.856 \\
\hline & Positive & $84.0 \pm 50.13$ & \\
\hline \multirow[t]{2}{*}{ Loss of appetite } & Negative & $73.11 \pm 35.73$ & 0.119 \\
\hline & Positive & $95.55 \pm 66.72$ & \\
\hline \multirow[t]{2}{*}{ ARDS } & Negative & $81.45 \pm 43.09$ & 0.859 \\
\hline & Positive & $83.94 \pm 56.87$ & \\
\hline \multirow[t]{2}{*}{ Distress } & Negative & $79.87 \pm 40.58$ & 0.779 \\
\hline & Positive & $84.17 \pm 55.82$ & \\
\hline \multirow[t]{2}{*}{ Renal pain } & Negative & $82.62 \pm 52.79$ & 0.833 \\
\hline & Positive & $87.80 \pm 46.29$ & \\
\hline \multirow[t]{2}{*}{ Shock } & Negative & $84.0 \pm 51.86$ & 0.001 \\
\hline & Positive & $26.19 \pm 11.19$ & \\
\hline \multirow[t]{2}{*}{ Anosmia } & Negative & $82.67 \pm 50.58$ & 0.866 \\
\hline & Positive & $86.5 \pm 68.96$ & \\
\hline \multirow[t]{2}{*}{ Pharyngitis } & Negative & $84.75 \pm 54.33$ & 0.753 \\
\hline & Positive & $80.41 \pm 49.11$ & \\
\hline \multirow[t]{2}{*}{ Agitation } & Negative & $85.23 \pm 53.33$ & 0.324 \\
\hline & Positive & $63.0 \pm 33.87$ & \\
\hline \multirow[t]{2}{*}{ Nausea } & Negative & $89.91 \pm 55.97$ & 0.030 \\
\hline & Positive & $63.75 \pm 32.75$ & \\
\hline \multirow[t]{2}{*}{ Diarrhea } & Negative & $85.06 \pm 54.46$ & 0.546 \\
\hline & Positive & $74.83 \pm 41.13$ & \\
\hline \multirow[t]{2}{*}{ Myalgia } & Negative & $83.92 \pm 41.61$ & 0.908 \\
\hline & Positive & $82.35 \pm 59.53$ & \\
\hline LOC & Negative & $84.33 \pm 52.98$ & 0.471 \\
\hline & Positive & $64.75 \pm 33.51$ & \\
\hline
\end{tabular}

Table 3 (continued)

\begin{tabular}{llll}
\hline Characteristics & Level & IGF-1 & $p$ value \\
\hline Chest pain & Negative & $84.19 \pm 53.68$ & 0.766 \\
& Positive & $79.53 \pm 47.88$ & \\
Fatigue & Negative & $84.51 \pm 50.77$ & 0.756 \\
& Positive & $80.05 \pm 55.55$ & \\
Weakness & Negative & $87.03 \pm 55.13$ & 0.415 \\
& Positive & $79.66 \pm 49.72$ & \\
Sputum & Negative & $82.15 \pm 50.55$ & 0.749 \\
& Positive & $88.22 \pm 62.65$ & \\
Headache & Negative & $87.92 \pm 57.43$ & 0.203 \\
& Positive & $72.26 \pm 36.16$ & \\
& &
\end{tabular}

The provided table represented a significant correlation between IGF-1 with hypertension, neurogenic diseases, shock, and Nausea in severe patients with COVID-19. Bold $p$ value indicated as statistically significant at the level of 0.05

$C K D$ Chronic kidney disease, COPD Chronic obstructive pulmonary disease, IHD Ischemic heart disease, ARDS Acute Respiratory Distress Syndrome, LOC Loss of Consciousness. Data are expressed as mean $\pm \mathrm{SD}$

Table 4 Correlation matrix between IGF-1 serum levels with BP, BPD, PR, RR, and $T$ in the severe COVID-19 patients

\begin{tabular}{llll}
\hline Group & Variable & Coefficient $(r)$ & $p$ value \\
\hline Severe patients with COVID-19 & BP & 0.015 & 0.970 \\
& BPD & 0.082 & 0.529 \\
& PR & 0.096 & 0.460 \\
& RR & 0.128 & 0.329 \\
& T & 0.017 & 0.899 \\
\hline
\end{tabular}

The supplied data demonstrated no remarkable differences between the serum levels of IGF-1 with BP, BPD, PR, RR, and T. Data correlation analysis by Spearman rank correlation. Blood Pressure Diastolic (BPD), Pulse Rate (PR), Respiratory Rate (RR), and Temperature $(T) . p<0.05$ was considered statistically significant.

Given the importance of aging as a critical risk factor in the pathogenesis of severe form of COVID-19, we evaluated the association between IGF-1 and age in patients and control groups. Although Secretion of IGF-1 drops over time with senescence (Junnila et al. 2013), there was a positive correlation between the IGF-1 serum levels and age in severely affected COVID-19 patients. In contrast, there was a negative association between the IGF-1 serum levels and age in healthy subjects. Aging is the main contributor to pulmonary physiology, function, and pathology during lung infection. Consequently, age-related differences may be associated with immune system responses and tolerance, leading to worse clinical outcomes in elderly individuals (Miller and Linge 2017). In addition, it has been 
demonstrated that age-related cellular and tissue function changes are associated with reducing the anabolic hormones, growth hormone, and IGF-1, which may lead to longevity (Sonntag et al. 2000, 2005; Shimokawa et al. 2003). Regarding the positive correlation between age and IGF-1 in severe COVID-19 patients, IGF-1 can be considered an inflammatory factor that may implicate the severity of COVID-19. Likewise, an increase in IGF-1 and IGFB-1 binding protein (IGFBP-3) was reported in the damage and death of lung epithelial cells, as well as early ARDS, while a decrease in IGFB-1and IGFBP-3 has been reported in late ARDS (Ahasic et al. 2015).

In the following, we investigated the correlation of IGF-1 and some crucial clinical characteristics in COVID19 patients. The IGF-1 levels were significantly higher in patients without hypertension, shock, and nausea than severe COVID-19 patients with hypertension, shock, and nausea. In line with our finding, various studies showed an inverse correlation between circulating IGF-1 levels and blood pressure among type 1 and type 2 diabetic patients and patients with borderline hypertension (Capoluongo et al. 2006; Sesti et al. 2005; Brismar 1998). Besides, another study revealed the serum levels of IGF-1 were remarkably declined in patients with septic shock compared to healthy subjects (Xu et al. 2017). Our data also showed a positive association between elevated IGF-1 levels with neurogenic diseases in patients with COVID-19.

Neurological complications are one of the essential findings in COVID-19. Several studies have demonstrated the elevated levels of IGF-1 in the central nervous system (CNS) following an injury, which may act as an endogenous protective mechanism (Kizhakke Madathil et al. 2010; Nordqvist et al. 1996). Regarding our finding and existing data about the role of IGF-1 in neurological disorders, it can be hypothesized that IGF-1 may contribute to the neurologic manifestation of COVID-19.

Considering the current and previous studies, the IGF-1 may have a role in the pathogenesis of severe form of COVID-19, and unraveling its exact contribution warrant further investigation.

\section{Conclusion}

In sum, our data imply the complex role of IGF-1 in COVID19 patients. Further studies are warranted to confirm our findings and elucidate underlying mechanisms.

Acknowledgements This work was supported by the Kermanshah University of Medical Sciences (Grant number 990599). We thank the deputy of Research and Technology of Kermanshah University of Medical Sciences for financial support.
Author contributions MT contributed to the idea design, checked the final results, and performed the final revision of the manuscript. ES performed the experiments and contributed to the idea design. SM provided patients samples and clinical data. ShM and SAR summarized and analyzed the data. PF drafted and wrote the manuscript. All authors reviewed and approved the final manuscript.

\section{Declarations}

Conflict of interest The authors have no conflict of interest and they alone are responsible for the content and writing of the paper.

\section{References}

Ahasic AM, Zhai R, Su L, Zhao Y, Aronis KN, Thompson BT, Mantzoros CS, Christiani DC (2012) IGF1 and IGFBP3 in the acute respiratory distress syndrome. Eur J Endocrinol Eur Federation Endocr Soc 166:121

Ahasic AM, Tejera P, Wei Y, Su L, Mantzoros CS, Bajwa EK, Thompson BT, Christiani DC (2015) Predictors of Circulating InsulinLike Growth Factor-1 and Insulin-Like Growth Factor Binding Protein-3 in Critical Illness. Crit Care Med 43:2651

Azkur AK, Akdis M, Azkur D, Sokolowska M, van de Veen W, Brüggen MC, O’Mahony L, Gao Y, Nadeau K, Akdis CA (2020) Immune response to SARS-CoV-2 and mechanisms of immunopathological changes in COVID-19. Allergy 75:1564-1581

Brismar CL (1998) KERSTIN: Insulin-like growth factor binding protein-1 as a marker of the metabolic syndrome-a study in borderline hypertension. Blood Press 7:88-95

Capoluongo E, Pitocco D, Lulli P, Minucci A, Santonocito C, Manto A, Di Stasio E, Zaccardi F, Zuppi C, Ghirlanda G (2006) Inverse correlation between serum free IGF-I and IGFBP-3 levels and blood pressure in patients affected with type 1 diabetes. Cytokine 34:303-311

DeBoer MD, Scharf RJ, Leite AM, Férrer A, Havt A, Pinkerton R, Lima AA, Guerrant RL (2017) Systemic inflammation, growth factors, and linear growth in the setting of infection and malnutrition. Nutrition 33:248-253

Delafontaine P, Song Y-H, Li Y (2004) Expression, regulation, and function of IGF-1, IGF-1R, and IGF-1 binding proteins in blood vessels. Arterioscler Thromb Vasc Biol 24:435-444

Fan X, Yin C, Wang J, Yang M, Ma H, Jin G, Song M, Hu Z, Shen H, Hang D (2020) Pre-diagnostic circulating concentrations of insulin-like growth factor-1 and risk of COVID-19 mortality: results from UK Biobank. Eur J Epidemiol 2021:1-8

He J, Mu M, Wang H, Ma H, Tang X, Fang Q, Guo S, Song C (2019) Upregulated IGF-1 in the lungs of asthmatic mice originates from alveolar macrophages. Mol Med Rep 19:1266-1271

Heemskerk VH, Daemen MA, Buurman WA (1999) Insulin-like growth factor-1 (IGF-1) and growth hormone (GH) in immunity and inflammation. Cytokine Growth Factor Rev 10:5-14

Hoshino M, Nakamura Y, Sim J, Yamashiro Y, Uchida K, Hosaka K, Isogai S (1998) Inhaled corticosteroid reduced lamina reticularis of the basement membrane by modulation of insulin-like growth factor (IGF)-I expression in bronchial asthma. Clin Exp Allergy $\mathbf{J}$ Br Soc Allergy Clin Immunol 28:568-577

Jogie-Brahim S, Feldman D, Oh Y (2009) Unraveling insulin-like growth factor binding protein-3 actions in human disease. Endocr Rev 30:417-437

Junnila RK, List EO, Berryman DE, Murrey JW, Kopchick JJ (2013) The GH/IGF-1 axis in ageing and longevity. Nat Rev Endocrinol $9: 366$ 
Kassir R (2020) Risk of COVID-19 for patients with obesity. Obesity Rev. https://doi.org/10.1111/obr.13034

Kizhakke Madathil S, Evans HN, Saatman KE (2010) Temporal and regional changes in IGF-1/IGF-1R signaling in the mouse brain after traumatic brain injury. J Neurotrauma 27:95-107

Krein PM, Sabatini PJ, Tinmouth W, Green FH, Winston BW (2003) Localization of insulin-like growth factor-I in lung tissues of patients with fibroproliferative acute respiratory distress syndrome. Am J Respir Crit Care Med 167:83-90

Li S, Geng J, Xu X, Huang X, Leng D, Jiang D, Liang J, Wang C, Jiang D, Dai H (2016) miR-130b-3p modulates epithelial-mesenchymal crosstalk in lung fibrosis by targeting IGF-1. PLoS ONE 11:e0150418

Li G, Zhou L, Zhang C, Shi Y, Dong D, Bai M, Wang R, Zhang C (2019) Insulin-like growth factor 1 regulates acute inflammatory lung injury mediated by influenza virus infection. Front Microbiol 10:2541

Li G, Fan Y, Lai Y, Han T, Li Z, Zhou P, Pan P, Wang W, Hu D, Liu $X$ (2020) Coronavirus infections and immune responses. J Med Virol 92:424-432

Martin-Estal I, De La Garza R, Castilla-Cortazar I (2016) Intrauterine growth retardation (IUGR) as a novel condition of insulin-like growth factor-1 (IGF-1) deficiency. Rev Physiol Biochem Pharmacol 170:1-35

Miller EJ, Linge HM (2017) Age-related changes in immunological and physiological responses following pulmonary challenge. Int J Mol Sci 18:1294

Mu M, Gao P, Yang Q, He J, Wu F, Han X, Guo S, Qian Z, Song C (2020) Alveolar epithelial cells promote IGF-1 production by alveolar macrophages through TGF- $\beta$ to suppress endogenous inflammatory signals. Front Immunol. https://doi.org/10.3389/ fimmu.2020.01585

Myers MG, Backer JM, Sun XJ, Shoelson S, Hu P, Schlessinger J, Yoakim M, Schaffhausen B, White MF (1992) IRS-1 activates phosphatidylinositol 3'-kinase by associating with src homology 2 domains of $\mathrm{p} 85$. Proc Natl Acad Sci 89:10350-10354

Narasaraju TA, Chen H, Weng T, Bhaskaran M, Jin N, Chen J, Chen Z, Chinoy MR, Liu L (2006) Expression profile of IGF system during lung injury and recovery in rats exposed to hyperoxia: a possible role of IGF-1 in alveolar epithelial cell proliferation and differentiation. J Cell Biochem 97:984-998

Nordqvist AC-S, Von Holst H, Holmin S, Sara V, Bellander B-M, Schalling M (1996) Increase of insulin-like growth factor (IGF)-1, IGF binding protein- 2 and -4 mRNAs following cerebral contusion. Mol Brain Res 38:285-293

Norling AM, Gerstenecker AT, Buford TW, Khan B, Oparil S, Lazar RM (2020) The role of exercise in the reversal of IGF-1 deficiencies in microvascular rarefaction and hypertension. GeroScience 42:141-158

of the International CSG (2020) The species Severe acute respiratory syndrome-related coronavirus: classifying 2019-nCoV and naming it SARS-CoV-2. Nat Microbiol 5:536

Ounis OB, Elloumi M, Zouhal H, Makni E, Denguezli M, Amri M, Lac G, Tabka Z (2010) Effect of individualized exercise training combined with diet restriction on inflammatory markers and IGF-1/IGFBP-3 in obese children. Ann Nutr Metab 56:260-266
Qin C, Zhou L, Hu Z, Zhang S, Yang S, Tao Y, Xie C, Ma K, Shang K, Wang W (2020) Dysregulation of immune response in patients with coronavirus 2019 (COVID-19) in Wuhan, China. Clin Infect Dis 71:762-768

Salminen A, Kaarniranta K (2010) Insulin/IGF-1 paradox of aging: regulation via AKT/IKK/NF- $\mathrm{BB}$ signaling. Cell Signal 22:573-577

Schnapp LM, Donohoe S, Chen J, Sunde DA, Kelly PM, Ruzinski J, Martin T, Goodlett DR (2006) Mining the acute respiratory distress syndrome proteome: identification of the insulin-like growth factor (IGF)/IGF-binding protein-3 pathway in acute lung injury. Am J Pathol 169:86-95

Sesti G, Sciacqua A, Cardellini M, Marini MA, Maio R, Vatrano M, Succurro E, Lauro R, Federici M, Perticone F (2005) Plasma concentration of IGF-I is independently associated with insulin sensitivity in subjects with different degrees of glucose tolerance. Diabetes Care 28:120-125

Shimokawa I, Higami Y, Tsuchiya T, Otani H, Komatsu T, Chiba T, Yamaza H (2003) Lifespan extension by reduction of the growth hormone-insulin-like growth factor-1 axis: relation to caloric restriction. FASEB J 17:1108-1109

Sinha P, Matthay MA, Calfee CS (2020) Is a "cytokine storm" relevant to COVID-19? JAMA Intern Med 180:1152-1154

Sonntag WE, Lynch C, Thornton P, Khan A, Bennett S, Ingram R (2000) The effects of growth hormone and IGF-1 deficiency on cerebrovascular and brain ageing. J Anat 197:575-585

Sonntag WE, Carter CS, Ikeno Y, Ekenstedt K, Carlson CS, Loeser RF, Chakrabarty S, Lee S, Bennett C, Ingram R (2005) Adultonset growth hormone and insulin-like growth factor I deficiency reduces neoplastic disease, modifies age-related pathology, and increases life span. Endocrinology 146:2920-2932

Spielman LJ, Little JP, Klegeris A (2014) Inflammation and insulin/ IGF-1 resistance as the possible link between obesity and neurodegeneration. J Neuroimmunol 273:8-21

Stiles AD, D'Ercole AJ (1990) The insulin-like growth factors and the lung. Am J Respir Cell Mol Biol 3:93-100

Team EE (2020) Note from the editors: World Health Organization declares novel coronavirus (2019-nCoV) sixth public health emergency of international concern. Eurosurveillance 25:200131e

Teppala S, Shankar A (2010) Association between serum IGF-1 and diabetes among US adults. Diabetes Care 33:2257-2259

Xu L, Zhang W, Sun R, Liu J, Hong J, Li Q, Hu B, Gong F (2017) IGF-1 may predict the severity and outcome of patients with sepsis and be associated with microRNA-1 level changes. Exp Ther Med 14:797-804

Ye Q, Wang B, Mao J (2020) The pathogenesis and treatment of theCytokine Storm'in COVID-19. J Infect 80:607-613

Zhu N, Zhang D, Wang W, Li X, Yang B, Song J, Zhao X, Huang B, Shi W, Lu R (2020) A novel coronavirus from patients with pneumonia in China, 2019. N Engl J Med 382:727

Publisher's Note Springer Nature remains neutral with regard to jurisdictional claims in published maps and institutional affiliations. 